International Journal of Current Advanced Research

ISSN: O: 2319-6475, ISSN: P: 2319 - 6505, Impact Factor: SJIF: 5.995

Available Online at www.journalijcar.org

Volume 6; Issue 5; May 2017; Page No. 3724-3727

DOI: http://dx.doi.org/10.24327/ijcar.2017.3727.0356

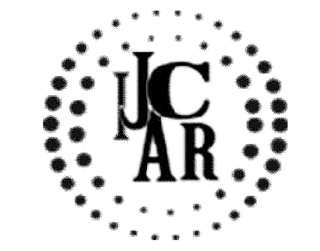

Review Article

\title{
NEWER DIAGNOSTIC TECHNIQUES IN TUBERCULOSIS- A REVIEW
}

\author{
Abhishek $\mathbf{N}$ and Gopinath $P$ \\ Saveetha Dental College, Chennai
}

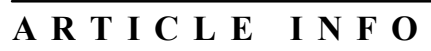

\section{Article History:}

Received $18^{\text {th }}$ February, 2017

Received in revised form $12^{\text {th }}$ March, 2017

Accepted $5^{\text {th }}$ April, 2017

Published online $28^{\text {th }}$ May, 2017

\section{Key words:}

Tuberculosis, Bactec460, Pcr, Quantiferon Tb Gold, Fastplaque Tb

\begin{abstract}
A B S T R A C T
Aim: To review about the newer diagnostic techniques of the tuberculosis

Objective: to provide information about various diagnostic techniques of tuberculosis disease

Background: Early confirmation of the diagnosis of tuberculosis is a challenging problem specially in case of paucibacillary and extra-pulmonary forms.Diagnosis of tuberculosis is mainly based on clinical features, histopathology, demonstration of acid fast bacilli (AFB) and isolation of Mycobacterium tuberculosis from the clinical specimens. These techniques have limitations of speed, sensitivity and specificity. During the last two decades several rapid techniques for detection of early growth have been described which can help in obtaining the culture and sensitivity reports relatively

Reason: to provide information about the various newer diagnostic techniques about tuberculosis
\end{abstract}

Copyright $@ 2017$ Abhishek $\boldsymbol{N}$ and Gopinath $\boldsymbol{P}$. This is an open access article distributed under the Creative Commons Attribution License, which permits unrestricted use, distribution, and reproduction in any medium, provided the original work is properly cited.

\section{INTRODUCTION}

Despite the discovery of the tuberculosis disease a hundred years ago and all the advances in our knowledge of the disease made till then yet tuberculosis remains one of the major problems facing mankind [1]. Due to limited advances in diagnosis of tuberculosis (TB) for more than a century, the development of new diagnostic tools has become a central part of the TB research in recent years. Many organizations have acknowledged the need for improved TB diagnostics, and have advocated for additional research [2]. Several TB diagnostic tests are currently under development and some are even under use in many countries. Unfortunately, tests that would have the greatest impact on TB control are only in early development stages and are put to little use in some countries. New diagnostic tests that would increase the sensitivity or simplicity of diagnosing active disease are in later stages of development but they are expected to have a measurable impact on TB control. Tuberculin skin testing is still the most common method in use, despite its shortcomings. Novel diagnostic methods for use in TB diagnostic studies are highly desirable.[3,4]. During the last few decades, several methods for achieving early diagnosis of tuberculosis have been developed.

This review examines the recent advances in the diagnosis of tuberculosis in humans.

*Corresponding author: Abhishek N

Saveetha Dental College, Chennai
Newer diagnostic techniques in tuberculosis are

\section{BACTEC 460TB}

BACTEC 460TB has been long considered the best method for rapid testing of susceptibility of $M$ tuberculosis to major anti-tuberculous drugs such as rifampicin, isoniazid, ethambutol, pyrazinamide and streptomycin in clinical laboratories [5] An Indian study showed that the BACTEC 460TB radiometric method obtained $87 \%$ of the positive results within seven days and $96 \%$ within 14 days [6,7] Therefore, by facilitating early diagnosis, the BACTEC 460TB method is considered cost effective in countries endemic for tuberculosis[6] This radiometric technique uses 14C labeled palmitic acid in $7 \mathrm{H} 12$ medium to detect the metabolism rather than the visible growth of mycobacteria in half the time required by conventional culture methods [8] The metabolism of the $14 \mathrm{C}$ labeled substrate leads to the production of $14 \mathrm{CO} 2$ which is measured and reported in terms of growth index.

\section{ESP Culture System II}

For growth and detection of mycobacteria ESP Culture System II is a fully automated continuous monitoring culture system used for the growth and detection of different microorganisms (including $M$ tuberculosis). This test interprets mycobacterial growth by evaluating gas consumption, which leads to changes in the pressure above the culture medium[9]. 


\section{TB PNA Fish}

Fluorescence in situ hybrid ization (FISH) using peptide nucleic acid (PNA) probes allows differentiation between tuberculous and nontuberculous mycobacteria in smears of mycobacterial cultures. PNA molecules are pseudopeptides with DNA-binding capacity [10] in which the sugar phosphate backbone of DNA has been replaced by a polyamide backbone. 11 The $\mathrm{M}$ tuberculosis complexspecific PNA probes showed sensitivities of $84-97 \%$ while the nontuberculous mycobacteria-specific PNA probes showed diagnostic sensitivities of $64-91 \%$. Both types of probes showed diagnostic specificities and predictive values of $100 \%$

\section{Xpert MTB/RIF}

Xpert MTB/RIF is an automated molecular test for $\mathrm{M}$ tuberculosis and its resistance to rifampin, based on the Cepheid GeneXpert system. It uses hemi-nested real-time PCR assay to amplify a specific sequence of the rpoB gene, which is then probed with molecular beacons for mutations within the rifampin-resistance determining region, providing a result within two hours[11].

\section{PCR}

PCR allows sequences of DNA to be amplified in vitro even when only a few copies of mycobacteria are present, so that the amount of amplified NDA can be rapidly visualized and identified. 11 The most common target used for PCR is insertion sequence IS6110.11,35 This sequence is specific for $M$ tuberculosis and offers multiple targets for amplification, being present up to 20 times in the genome. $11 \mathrm{~A}$ recent study performed in Bangladesh reported the sensitivity and specificity of PCR using the IS6110 to be $94.74 \%$ and $100 \%$ respectively, concluding that the PCR technique is a rapid and alternative method to LöwensteinJensen culture for the diagnosis of pulmonary tuberculosis [12]

\section{QuantiFERON-TB Gold}

QuantiFERON-TB Gold is an ELISA test which detects the release of IFN-gamma in fresh heparinized whole blood from sensitized persons upon incubation with synthetic peptides simulating ESAT-6 and culture filtrate protein-10 (CFP-10)[13]. The test steps for QuantiFERON-TB Gold involve blood sample collection, addition of stimulating antigens, incubation for $16-24$ hours at $37^{\circ} \mathrm{C}$, harvesting of plasma and addition of conjugate solution. The samples are then incubated for two hours at room temperature, the plates are washed at least six times and then the substrate is added. The samples are then incubated for 30 minutes, adding stop solution, reading absorbance at $450 \mathrm{~nm}$ and calculating results using dedicated software. The patient only needs to visit once, for specimen collection, and results can be obtained in 48 hours.

\section{QuantiFERON-TB Gold In-Tube}

The QuantiFERON-TB Gold In-Tube was developed to overcome the limitation of QuantiFERON-TB Gold, which could only be used in facilities where blood testing could begin within a few hours of its collection. This test uses a mixture of 14 peptides representing ESAT-6, CFP-10 and a part of TB7.7.[13]

T-SPOT.TB
T-SPOT.TB incubates peripheral blood mononuclear cells with mixtures of peptides (ESAT-6, CFP-10) and uses an enzyme-linked immunospot assay (ELISpot) to detect increases in the number of cells that secrete IFN-gamma (spots in each test well). 53 A recent study has compared QuantiFERONTB Gold In-Tube, T-SPOT.TB and tuberculin skin test (TST) in $373 \mathrm{HIV}$-infected patients, $[14,15]$ reporting that IGRAs were more sensitive than TST for the diagnosis of $\mathrm{M}$ tuberculosis infection in this category of patients. Ramos et al. propose dual sequential testing with both TST and IGRAs as the optimal approach for latent tuberculosis infection screening in this population.[16]

\section{FAST Plaque TB}

This is an original phage based test, which uses the mycobacteriophage to detect the presence of M.tuberculosis directly from sputum specimens. It is a rapid, manual test, easy to perform and has an overall higher sensitivity when compared with sputum smear microscopy, in newly diagnosed smear positive TB patients. The test has a specificity of 98.7 $99.0 \%$ and a sensitivity of $70.3-75.2 \%$, when compared with smear microscopy, which has a specificity of $97.3-97.4 \%$ and a sensitivity of $61.3-63.4 \%[17,18]$

\section{MGIT 960 mycobacteria detection system}

It is an automated system for the growth and detection of mycobacteria with a capacity to incubate and continuously monitor 960 mycobacteria growth indicator tube (MGIT) every 60 minutes for increase in fluorescence. Growth detection is based on the AFB metabolic $\mathrm{O} 2$ utilization and subsequent intensification of an $\mathrm{O} 2$ quenched fluorescent dye contained in a tube of modified MGIT. A series of algorithms are used to determine presumptive positivity and alert the operator to the presence and location of positive tubes.

In a multicentre evaluation of the MGIT 960 system, three high volume testing sites in USA compared the growth and recovery of mycobacteria to that of BACTEC $460 \mathrm{~TB}$ and conventional culture methods 16 . Comparison of average time of detection between paired specimens showed that, the BACTEC 460 and MGIT 960 systems were 8.7 versus 8.6 days for M.avium complex (MAC) and 13.4 versus 15.5 days for M.tuberculosis respectively. It was revealed that MGIT 960 system exhibits greater potential as a rapid, accurate and cost effective method for a high volume mycobacteriology laboratory [19]

\section{Septi-check AFB method}

The septi-chek AFB system consists of a capped bottle containing $30.0 \mathrm{ml}$ of middle-brook $7 \mathrm{H} 9$ broth under enhanced (5-8\%) $\mathrm{CO} 2$, a paddle with agar media enclosed in a plastic tube, and enrichment broth containing glucose, glycerin, oleic acid, pyridoxal, catalase, albumin, polyoxyethylene 40 stearate, azlocillin, nalidixic acid, trimethoprim, polymyxin B and amphotericin B. One side of the paddle is covered with non-selective middle brook $7 \mathrm{H} 11$ agar, the reverse side is divided into two sections: one contains 7H11 agar with para-nitro-a-acetylamino-bhydroxypropiophenone (NAP) for differentiation of M.tuberculosis from other mycobacteria, the other section contains chocolate agar for detection of contaminants. This non-radiometric approach has the potential to expedite processing, obviate $\mathrm{CO} 2$ incubation requirements and 
facilitates early detection of positive cultures. This method requires about 3 weeks of incubation. The unique advantage of this technique is the simultaneous detection of M.tuberculosis, non-tuberculous mycobacteria (NTM), other respiratory pathogens and even contaminants. A multicentric study conducted in the USA has shown that the system gives a better culture result when compared to other methods including BACTEC 460 TB system [20].

\section{TB MPB 64 patch test}

MPB 64 is a specific mycobacterial antigen for M.tuberculosis complex. This patch test becomes positive in 3-4 days after patch application and lasts for a week. The test has a specificity of $100 \%$ and a sensitivity of $98.1 \%$ [21]. This promising test has been reported so far only in one setting in Philippines and need to be carried out in other settings.

Another approach is the use of defined antigens for an accurate and rapid test for tuberculosis infection based on the detection of $\mathrm{T}$ cells sensitized to M.tuberculosis either by blood tests in vitro or skin tests in vivo [22]. Mononuclear cells from the peripheral blood are stimulated in vitro and production of IFN gamma from the sensitized $\mathrm{T}$ cells is measured by ELISA [23]. The antigens used are ESAT 6 (early secretory antigen TB) and CFP 10 (colony forming protein), which are being used as an alternative for PPD, for use in skin test (tuberculin testing) in vivo.

\section{CONCLUSION}

In conclusion, the advantages and disadvantages of each available TB diagnostic method are evident and no test is yet available to meet target specification in terms of performance and ease of use. Apart from these characteristics of current TB diagnostics, the quality of test results with the existing methods is dependent on the availability of sufficient human and financial resources, training of laboratory personnel and monitoring of performance. New methods that manage to overcome limitations and respond to challenges posed by special populations will definitely be well received.

\section{References}

1. Newer diagnostic techniques for tuberculosis.

2. Katoch VM1. PMID: 15520490

3. Siddiqi K, Lambert ML, Walley J. Clinical diagnosis of smear-negative pulmonary tuberculosis in lowincome countries: the current evidence. Lancet Infect Dis. 2003; 3(5): 288-96.

4. Xue Y, Bai Y, Gao X. Expression, purification and characterization of Mycobacterium tuberculosis RpfE protein. J Biomed Res. 2012; 26(1): 17-23.

5. Baths V, Roy U. Mycobacterium tuberculosis using statistical coupling analysis of the esterase family proteins. J Biomed Res. 2011; 25(3): 165-9.

6. Scarparo C, Ricordi P, Ruggiero G, Piccoli P. Evaluation of the fully automated BACTEC MGIT 960 system for testing susceptibility of Mycobacterium tuberculosis to pyrazinamide, streptomycin, isoniazid, rifampin, and ethambutol and comparison with the radiometric BACTEC 460TB method. J Clin Microbiol. 2004; 42(3): 110914.
7. Ramachandran R, Paramasivan C. What is new in the diagnosis of tuberculosis? Part 1: Techniques for diagnosis of tuberculosis. Ind J Tub. 2003; (50): 13341.

8. Venkataraman P, Herbert D, Paramasivan CN. Evaluation of the BACTEC radiometric method in the early diagnosis of tuberculosis. Indian $\mathrm{J} \mathrm{Med}$ Res. 1998; 108: 120-7.

9. Conventional culture methods. 17 The metabolism of the $14 \mathrm{C}$ labeled substrate leads to the production of $14 \mathrm{CO} 2$ which is measured and reported in terms of growth index.

10. Sh G, Plaunt M, Murphy T. Clinical evaluation of difco ESP culture system II for growth and detection of mycobacteria. J Clin Microbiol. 1997; 35(1): 121-4.

11. Stender H, Lund K, Petersen KH, Rasmussen OF, Hongmanee $\mathrm{P}$, Miorner $\mathrm{H}$, et al. Fluorescence In situ hybridization assay using peptide nucleic acid probes for differentiation between tuberculous and nontuberculous mycobacterium species in smears of mycobacterium cultures. J Clin Microbiol. 1999; 37(9): 2760-5.

12. Boehme CC, Nabeta P, Hillemann D, Nicol MP, Shenai S, Krapp F, et al. Rapid molecular detection of tuberculosis and rifampin resistance. $N$ Engl J Med. 2010; 363(11): 1005-15.

13. Hasan MM, Hossain MA, Paul SK, Mahmud C, Khan ER, Rahman MM, et al. Evaluation of PCR with culture for the diagnosis of pulmonary tuberculosis. Mymensingh Med J. 2012; 21(3): 399403.

14. Mazurek GH, Jereb J, Vernon A, LoBue P, Goldberg S, Castro K. Updated guidelines for using Interferon Gamma Release Assays to detect Mycobacterium tuberculosis infection - United States, 2010. MMWR Recomm Rep. 2010; 59(RR-5): 125.

15. Aramă V, Tilişcan C, Ion D, Mihăilescu R, Munteanu D, Streinu-Cercel A, et al. Serum adipokines and HIV viral replication in patients undergoing antiretroviral therapy. GERMS. 2012; 2(1): 12-7.

16. Uaraldi G. Evolving approaches and resources for clinical practice in the management of HIV infection in the HAART era. GERMS. 2011; 1(1).

17. Ramos JM, Robledano C, Masia M, Belda S, Padilla S, Rodriguez JC, et al. Contribution of Interferon gamma release assays testing to the diagnosis of latent tuberculosis infection in HIVinfected patients: A comparison of QuantiFERON-TB gold in tube, T-SPOT.TB and tuberculin skin test. BMC Infect Dis. 2012; 12(1): 169.h.

18. Albert, H., Heydenrych, A., Brookes, R., Mole, R.J., Harley, B., Subotsky, E., Henry, R. and Azevedo, V. Performance of a rapid phage-based test, FAST Plaque $\mathrm{TB}^{\mathrm{TM}}$ to diagnose pulmonary tuberculosis from sputum specimens in South Africa. Int J Tuberc Lung Dis 6: 529, 2002.

19. Muzaffar., R., Batool, S., Aziz, F., Naqvi, A. and Rizvi, R. Evaluation of FAST Plaque TB assay for 
direct detection of M.tuberculosis in sputum specimens. Int J Tuberc Lung Dis 6: 635, 2002.

20. Hanna BA1, Ebrahimzadeh A, Elliott LB, Morgan MA, Novak SM, Rusch-Gerdes S, Acio M, Dunbar DF, Holmes TM, Rexer CH, Savthyakumar C, Vannier AM. Multicenter evaluation of the BACTEC MGIT 960 system for recovery of mycobacteria

21. Isenberg, H.D., D’ Amato, R.F., Heifets, L., Murray, P.R., Scardamaglia, M., Jacobs, M.C., Alperstein, P. and Niles, A. Collaborative feasibility study of a biphasic system (Roche Septi-Chek AFB) for rapid detection and isolation of Mycobacteria. $J$ Clin Microbiol 29: 1719, 1991
22. Nakamura,R.M.,Velmonte,M.A.,Kawajiri,K.,Ang,C.F., Frias,R.A., Mendoza, M.T., Montoya, J.C., Honda, I., Haga, S. and Toida, I. MPB 64 mycobacterial antigen: A new skin-test reagent through patch method for rapid diagnosis of active tuberculosis. Int J Tuberc Lung Dis 2: $541,1998$.

23. Anderson, P., Munk, M.E., Pollock, J.M. and Doharty, T.M. Specific immune-based diagnosis of tuberculosis. Lancet 356: 1099, 2000.

24. Streeton, J.A., Deseim, N. and Jones, S.L. Sensitivity and specificity of a gamma interferon blood test for tuberculosis infection. In J Tuberc Lung Dis 2: 443, 1998.

\section{How to cite this article:}

Abhishek N and Gopinath P (2017) 'Newer Diagnostic Techniques In Tuberculosis- A Review', International Journal of

Current Advanced Research, 06(05), pp. 3724-3727.

DOI: http://dx.doi.org/10.24327/ijcar.2017.3727.0356 